PROCEEDINGS OF THE

AMERICAN MATHEMATICAL SOCIETY

Volume 131, Number 2, Pages 479-485

S 0002-9939(02)06537-1

Article electronically published on June 3, 2002

\title{
HYPERCYCLICITY AND SUPERCYCLICITY FOR INVERTIBLE BILATERAL WEIGHTED SHIFTS
}

\author{
NATHAN S. FELDMAN
}

(Communicated by Joseph A. Ball)

\begin{abstract}
We give a characterization of the invertible bilateral weighted shifts that are hypercyclic or supercyclic. Although there is a general characterization due to H. Salas, in the invertible case the conditions simplify greatly.
\end{abstract}

\section{INTRODUCTION}

A linear operator $T$ on a Hilbert space $\mathcal{H}$ is hypercyclic if there is a vector with dense orbit; that is, if there exists an $x \in \mathcal{H}$ such that $\left\{x, T x, T^{2} x, \ldots\right\}$ is dense in $\mathcal{H}$. An operator $T$ is supercyclic if there exists a vector whose scaled orbit is dense; that is, if there exists an $x \in \mathcal{H}$ such that $\left\{\lambda T^{n} x: n \geq 0, \lambda \in \mathbb{C}\right\}$ is dense in $\mathcal{H}$. The first example of a hypercyclic operator on a Banach space was a multiple of the backward shift on $\ell^{2}(\mathbb{N})$; it was shown in 1969 by Rolewicz [6] that if $B$ is the backward shift, then $\lambda B$ is hypercyclic if and only if $|\lambda|>1$. It then follows easily that $B$ itself is supercyclic. It was shown later in 1974 by Hilden and Wallen [4] that any (unilateral) backward weighted shift is supercyclic. These results were fairly constructive and particular to weighted shifts.

In 1982 Kitai [5] in her thesis and, independently, Gethner and Shapiro [3] gave a criterion that implies hypercyclicity for more general operators; it has become known as the "Hypercyclicity Criterion". The Hypercyclicity Criterion has been widely used to show that many different types of operators are hypercyclic. For instance hypercyclic operators arise in the classes of composition operators [1, adjoints of multiplication operators [3], cohyponormal operators [2], and weighted shifts 7].

In 7] and [8, Salas characterized the bilateral weighted shifts that are hypercyclic and those that are supercyclic in terms of their weight sequence. However the characterization is (necessarily) rather complicated, involving several quantifiers.

In this paper the author, in an attempt to verify Salas' characterization for a particular example, found that a much simpler condition characterizes the invertible bilateral weighted shifts that are hypercyclic or supercyclic. However, we give an example showing that this simpler condition does not give a characterization in general.

We first give our characterization in the invertible case and then present an example showing that the simpler condition is not always sufficient. Finally we

Received by the editors April 28, 2001 and, in revised form, September 17, 2001.

2000 Mathematics Subject Classification. Primary 47A16, 47B37.

Key words and phrases. Hypercyclic, supercyclic, bilateral weighted shift. 
show that one may relax the invertibility hypothesis somewhat and only assume that the negative (or positive) weights are bounded below.

\section{Preliminaries}

The principal tools used to show that operators are hypercyclic or supercyclic are the following criteria. Below is a variation of the hypercyclicity criterion due independently to Kitai [5] and Gethner and Shapiro [3].

Theorem 2.1 (Hypercyclicity Criterion). Suppose that $T \in \mathcal{L}(X)$. If there exist two dense sets $Y$ and $Z$ in $X$ and a sequence $n_{k} \rightarrow \infty$ such that:

(1) $T^{n_{k}} x \rightarrow 0$ for every $x \in Y$, and

(2) there exists a function $B: Z \rightarrow Z$ such that $T B x=x$ for all $x \in Z$ and $B^{n_{k}} x \rightarrow 0$ for every $x \in Z$,

then $T$ is hypercyclic.

Salas [8] developed a criterion for proving that certain operators are supercyclic. A more general supercyclicity criteria was given in Feldman, Miller and Miller [2].

Theorem 2.2 (A Supercyclicity Criterion). Suppose that $T \in \mathcal{L}(X)$. If there exist a sequence $n_{k} \rightarrow \infty$ and two dense sets $Y$ and $Z$ such that:

(1) there exists a function $B: Z \rightarrow Z$ such that $T B x=x$ for all $x \in Z$, and

(2) if $y \in Y$ and $x \in Z$, then $\left\|T^{n_{k}} x\right\|\left\|B^{n_{k}} y\right\| \rightarrow 0$ as $n \rightarrow \infty$,

then $T$ is supercyclic.

Note that the functions $B$, which are right inverses of $T$, need only be well defined maps; they may be, and usually are, discontinuous.

Next we give the characterizations of hypercyclicity and supercyclicity for weighted shifts as given by Salas [7, 8].

Theorem 2.3 (Hector Salas, 1995). Let T be a bilateral weighted shift with positive weight sequence $\left\{w_{n}\right\}$. Then $T$ is hypercyclic if and only if for any $\epsilon>0$ and $q \in \mathbb{N}$, there exists an arbitrarily large $n$, such that for all $j \in \mathbb{Z}$ with $|j| \leq q$, we have

$$
\prod_{s=0}^{n-1} w_{s+j}<\epsilon \quad \text { and } \quad \prod_{s=1}^{n} \frac{1}{w_{j-s}}<\epsilon .
$$

Theorem 2.4 (Hector Salas, 1999). Let T be a bilateral weighted shift with positive weight sequence $\left\{w_{n}\right\}$. Then $T$ is supercyclic if and only if for every $q \in \mathbb{N}$,

$$
\liminf _{n \rightarrow \infty} \max \left\{\frac{\prod_{k=j}^{j+n-1} w_{k}}{\prod_{k=h-1}^{h-n} w_{k}}:|j|,|h| \leq q\right\}=0 .
$$

\section{INVERTIBLE HYPERCYCLIC AND SUPERCYCLIC WEIGHTED SHIFTS}

We now present a simpler condition for an invertible bilateral weighted shift to be hypercyclic or supercyclic. Our proof is independent of Salas' work and makes use of the Hypercyclicity and Supercyclicity criteria.

Suppose $T$ is a bilateral weighted shift on $\ell^{2}(\mathbb{Z})$ with positive weights $\left\{w_{n}\right\}$. Let $\left(e_{n}\right)$ be the standard basis for $\ell^{2}(\mathbb{Z})$. Then $T$ acts on basis elements as follows: $T\left(e_{n}\right)=w_{n} e_{n+1}$ for all $n \in \mathbb{Z}$. We may define a right inverse $B$ to $T$ as follows: $B\left(e_{n}\right)=\frac{1}{w_{n-1}} e_{n-1}$. Then extend $B$ linearly to all vectors with finite support. 
Notice that $T B e_{n}=e_{n}$ for all $n$. If $T$ is invertible, then $B=T^{-1}$. Also observe that for each $n \in \mathbb{Z}$ and $k \geq 0$, we have

$$
T^{k}\left(e_{n}\right)=\left(\prod_{j=n}^{n+k-1} w_{j}\right) e_{n+k} \quad \text { and } \quad B^{k}\left(e_{n}\right)=\left(\prod_{j=n-1}^{n-k} \frac{1}{w_{j}}\right) e_{n-k} .
$$

Keeping the above notation, we now prove our characterizations. It follows easily from Salas' work that our conditions are necessary. We will prove that they are sufficient. The following lemma is the key point where invertibility is used.

Lemma 3.1. Suppose that $T$ is an invertible bilateral weighted shift and $\left\{n_{k}\right\}$ is a sequence of positive integers such that $n_{k} \rightarrow \infty$. If there exists an $n \in \mathbb{Z}$ such that $T^{n_{k}} e_{n} \rightarrow 0$ as $k \rightarrow \infty$, then $T^{n_{k}} e_{j} \rightarrow 0$ for all $j \in \mathbb{Z}$.

Proof. If $T^{n_{k}} e_{n} \rightarrow 0$ as $k \rightarrow \infty$ and $j \in \mathbb{Z}$, then there exist a $p \in \mathbb{Z}$ and $a>0$ such that $a T^{p}\left(e_{n}\right)=e_{j}$. Since $T^{p}$ is continuous and $T^{n_{k}}\left(e_{n}\right) \rightarrow 0$ we get $T^{n_{k}}\left(e_{j}\right)=$ $T^{n_{k}}\left(a T^{p}\left(e_{n}\right)\right)=a T^{p}\left(T^{n_{k}}\left(e_{n}\right)\right) \rightarrow 0$ as $k \rightarrow \infty$.

The invertibility is needed above because if $j<n$, then $p<0$, in which case we are using the existence and continuity of $T^{-1}$.

Since $T$ is invertible in the following arguments, $B=T^{-1}$ so that $B$ is also a bilateral weighted shift and as such Lemma 3.1 applies to it as well. We will use the notation $B$ for $T^{-1}$ simply to make an easy connection with the hypercyclicity criterion; also $B$ is of interest for non-invertible weighted shifts where $B$ is still well defined on vectors with finite support.

Theorem 3.2. If $T: \ell^{2}(\mathbb{Z}) \rightarrow \ell^{2}(\mathbb{Z})$ is an invertible bilateral weighted shift with weight sequence $\left(w_{n}\right)_{n=-\infty}^{\infty}$, then $T$ is hypercyclic if and only if there exists a sequence of integers $n_{k} \rightarrow \infty$ such that

$$
\lim _{k \rightarrow \infty} \prod_{j=1}^{n_{k}} w_{j}=0 \quad \text { and } \quad \lim _{k \rightarrow \infty} \prod_{j=1}^{n_{k}} \frac{1}{w_{-j}}=0 .
$$

Proof. Suppose the condition on the weights is satisfied. We will verify the Hypercyclicity Criterion with $Y=Z=\left\{x \in \ell^{2}(\mathbb{Z}): x\right.$ has only finitely many non-zero coordinates $\}$. Thus we need to show that if $x$ is any vector with finite support, then $T^{n_{k}} x \rightarrow 0$ and $B^{n_{k}} x \rightarrow 0$. It suffices to assume that $x=e_{n}$ for $n \in \mathbb{Z}$.

Furthermore, by Lemma 3.1 it suffices to show that $T^{n_{k}} e_{1} \rightarrow 0$ and $B^{n_{k}} e_{0} \rightarrow 0$. But that is easy because $\left\|T^{n_{k}}\left(e_{1}\right)\right\|=\prod_{j=1}^{n_{k}} w_{j} \rightarrow 0$ and $\left\|B^{n_{k}}\left(e_{0}\right)\right\|=\prod_{j=1}^{n_{k}} \frac{1}{w_{-j}} \rightarrow$ 0 .

Lemma 3.3. Suppose that $T$ is an invertible bilateral weighted shift and $\left\{n_{k}\right\}$ is a sequence of positive integers such that $n_{k} \rightarrow \infty$. If there exists $n, m \in \mathbb{Z}$ such that $\left\|T^{n_{k}} e_{n}\right\|\left\|B^{n_{k}} e_{m}\right\| \rightarrow 0$ as $k \rightarrow \infty$, then $\left\|T^{n_{k}} e_{i}\right\|\left\|B^{n_{k}} e_{j}\right\| \rightarrow 0$ for all $i, j \in \mathbb{Z}$.

Proof. If $i, j \in \mathbb{Z}$, then there exist $p, q \in \mathbb{Z}$ and $a, b>0$ such that $e_{i}=a T^{p}\left(e_{n}\right)$ and $e_{j}=b T^{q}\left(e_{q}\right)$. Thus

$$
\begin{aligned}
& \left\|T^{n_{k}}\left(e_{i}\right)\right\|\left\|B^{n_{k}}\left(e_{j}\right)\right\|=\left\|T^{n_{k}}\left(a T^{p}\left(e_{n}\right)\right)\right\|\left\|B^{n_{k}}\left(b T^{q}\left(e_{m}\right)\right)\right\| \\
& \quad \leq a b\left\|T^{p}\right\|\left\|T^{q}\right\|\left\|T^{n_{k}}\left(e_{n}\right)\right\|\left\|B^{n_{k}}\left(e_{m}\right)\right\| \rightarrow 0 \text { as } k \rightarrow \infty .
\end{aligned}
$$


Theorem 3.4. If $T: \ell^{2}(\mathbb{Z}) \rightarrow \ell^{2}(\mathbb{Z})$ is an invertible bilateral weighted shift with weight sequence $\left(w_{n}\right)_{n=-\infty}^{\infty}$, then $T$ is supercyclic if and only if there exists a sequence of integers $n_{k} \rightarrow \infty$ such that

$$
\lim _{k \rightarrow \infty}\left(\prod_{j=1}^{n_{k}} w_{j}\right)\left(\prod_{j=1}^{n_{k}} \frac{1}{w_{-j}}\right)=0
$$

Proof. We will verify that $T$ satisfies the supercyclicity criteria. Let $Y=Z=$ $\left\{x \in \ell^{2}(\mathbb{Z}): x\right.$ has only finitely many non-zero coordinates $\}$. We need to show that $\left\|T^{n_{k}} x\right\|\left\|B^{n_{k}} y\right\| \rightarrow 0$ as $k \rightarrow \infty$ for any $x, y \in Y$. It suffices, by linearity and the triangle inequality, to suppose that $x=e_{n}$ and $y=e_{m}$ for $n, m \in \mathbb{Z}$. However, by Lemma 3.3 it suffices to suppose that $x=e_{1}$ and $y=e_{0}$. But notice that $\left\|T^{n_{k}}\left(e_{1}\right)\right\|\left\|B^{n_{k}}\left(e_{0}\right)\right\|=\left(\prod_{j=1}^{n_{k}} w_{j}\right)\left(\prod_{j=1}^{n_{k}} \frac{1}{w_{-j}}\right) \rightarrow 0$ as $k \rightarrow \infty$. The necessity of this condition follows easily from Salas' characterization, Theorem 2.4 .

We now give an example of a non-invertible bilateral weighted shift that is not hypercyclic yet satisfies the condition in Theorem 3.2 . A similar example can be given for the condition in Theorem 3.4.

Example 3.5. If $T$ is the bilateral weighted shift with the weight sequence given below, then $T$ is not hypercyclic, but $T$ satisfies the following condition: There exists a sequence $\left\{n_{k}\right\}$ such that $\lim _{k \rightarrow \infty} \prod_{j=1}^{n_{k}} w_{j}=0$ and $\lim _{k \rightarrow \infty} \prod_{j=1}^{n_{k}} \frac{1}{w_{-j}}=0$. Thus for non-invertible operators Theorem 3.2 is not sufficient-it is only necessary.

\begin{tabular}{|c||c|c|c|c|c|c|c|c|c|c|c|c|c|c|c|c|c|}
\hline$n$ & -16 & -15 & -14 & -13 & -12 & -11 & $-10^{*}$ & -9 & -8 & -7 & -6 & $-5^{*}$ & -4 & -3 & $-2^{*}$ & -1 & 0 \\
\hline$w_{n}$ & 2 & 2 & 2 & 1 & 1 & $\frac{1}{2^{3}}$ & 2 & 2 & 2 & 1 & $\frac{1}{2^{2}}$ & 2 & 2 & $\frac{1}{2}$ & 2 & 1 & 1 \\
\hline
\end{tabular}

\begin{tabular}{|c||c|c|c|c|c|c|c|c|c|c|c|c|c|c|c|c|c|c|c|}
\hline$n$ & 1 & $2^{*}$ & 3 & 4 & $5^{*}$ & 6 & 7 & 8 & 9 & $10^{*}$ & 11 & 12 & 13 & 14 & 15 & 16 & $17^{*}$ & 18 & 19 \\
\hline$w_{n}$ & 1 & $\frac{1}{2}$ & 2 & 1 & $\frac{1}{2^{2}}$ & 2 & 2 & 1 & 1 & $\frac{1}{2^{3}}$ & 2 & 2 & 2 & 1 & 1 & 1 & $\frac{1}{2^{4}}$ & 2 & 2 \\
\hline
\end{tabular}

\begin{tabular}{|c||c|c|c|c|c|c|c|c|c|c|c|c|c|c|c|c|c|c|c|}
\hline$n$ & 1 & $2^{*}$ & 3 & 4 & $5^{*}$ & 6 & 7 & 8 & 9 & $10^{*}$ & 11 & 12 & 13 & 14 & 15 & 16 & $17^{*}$ & 18 & 19 \\
\hline$p_{n}$ & 1 & $\frac{1}{2}$ & 1 & 1 & $\frac{1}{2^{2}}$ & $\frac{1}{2}$ & 1 & 1 & 1 & $\frac{1}{2^{3}}$ & $\frac{1}{2^{2}}$ & $\frac{1}{2}$ & 1 & 1 & 1 & 1 & $\frac{1}{2^{4}}$ & $\frac{1}{2^{3}}$ & $\frac{1}{2^{2}}$ \\
\hline$q_{n}$ & 1 & 2 & 1 & 2 & $2^{2}$ & 1 & 1 & 2 & 4 & $2^{3}$ & 1 & 1 & 1 & 2 & 4 & 8 & $2^{4}$ & 1 & 1 \\
\hline
\end{tabular}

In the table above, $p_{n}=\prod_{j=1}^{n} w_{j}$ and $q_{n}=\prod_{j=1}^{n} w_{-j}$.

We claim that there is a weight sequence $\left\{w_{n}\right\}$ (as shown above) such that the following conditions hold:

(1) There exists a sequence $n_{k} \rightarrow \infty$ such that $p_{n_{k}} \rightarrow 0$ and $q_{n_{k}} \rightarrow \infty$, and

(2) for $n \geq 1$, we have $p_{n}<1$ and $q_{n}>1$ if and only if $n=n_{k}$ for some $k$.

In the example above the "starred" terms in the chart correspond to the sequence $\left\{n_{k}\right\}$ and we have that $p_{n_{k}}=\frac{1}{2^{k}}$ and $q_{n_{k}}=2^{k}$ and for any $n \notin\left\{n_{k}\right\}$, we have either $p_{n}=1$ or $q_{n}=1$.

\section{RELAXING INVERTIBILITY}

In this section we show that the assumption of invertibility can be relaxed in Theorems 3.2 and 3.4. Keeping the notation from the previous section, one easily sees that $T$ is invertible if and only if there exists $m>0$ such that $\left|w_{n}\right| \geq m$ for all $n \in \mathbb{Z}$. In this section we will show that Theorems 3.2 and 3.4 still hold with only 
the assumption that there exists an $m>0$ such that $w_{n} \geq m$ for all $n<0$ (or for all $n>0)$.

Theorem 4.1. Suppose that $T: \ell^{2}(\mathbb{Z}) \rightarrow \ell^{2}(\mathbb{Z})$ is a bilateral weighted shift with weight sequence $\left(w_{n}\right)_{n=-\infty}^{\infty}$ and $w_{n} \geq m>0$ for all $n<0$. Then:

(1) $T$ is hypercyclic if and only if there exists a sequence of integers $n_{k} \rightarrow \infty$ such that $\lim _{k \rightarrow \infty} \prod_{j=1}^{n_{k}} w_{j}=0$ and $\lim _{k \rightarrow \infty} \prod_{j=1}^{n_{k}} \frac{1}{w_{-j}}=0$.

(2) $T$ is supercyclic if and only if there exists a sequence of integers $n_{k} \rightarrow \infty$ such that $\lim _{k \rightarrow \infty}\left(\prod_{j=1}^{n_{k}} w_{j}\right)\left(\prod_{j=1}^{n_{k}} \frac{1}{w_{-j}}\right)=0$.

Proof. (1) Assume that there exists a sequence of integers $n_{k} \rightarrow \infty$ such that $\lim _{k \rightarrow \infty} \prod_{j=1}^{n_{k}} w_{j}=0$ and $\lim _{k \rightarrow \infty} \prod_{j=1}^{n_{k}} \frac{1}{w_{-j}}=0$. We will verify the conditions in Salas' characterization, Theorem 2.3. So, let $\epsilon>0$ and $q \in \mathbb{N}$. Suppose $\delta>0$ (we will prescribe $\delta$ later); then there exists an (arbitrarily large) $n_{k}$ such that $\prod_{j=1}^{n_{k}} w_{j}<\delta$ and $\prod_{j=1}^{n_{k}} \frac{1}{w_{-j}}<\delta$. Now let $n=n_{k}+q+1$ (this choice of $n$ guarantees that $n+j-1 \geq n_{k}$ for all $j$ with $\left.|j| \leq q\right)$. Then for $j \in \mathbb{Z}$ with $|j| \leq q$ we have

$$
\begin{aligned}
\prod_{s=0}^{n-1} w_{s+j} & =\prod_{s=j}^{n+j-1} w_{s}=C_{j}\left(\prod_{s=1}^{n_{k}} w_{s}\right)\left(\prod_{s=n_{k}+1}^{n+j-1} w_{s}\right) \\
& \leq C_{j}\left\|T^{2 q}\right\|\left(\prod_{s=1}^{n_{k}} w_{s}\right)<C_{j}\left\|T^{2 q}\right\| \delta,
\end{aligned}
$$

where $C_{j}=\left(\prod_{s=1}^{j-1} \frac{1}{w_{s}}\right)$ if $1<j \leq q, C_{j}=1$ if $j=1$, and $C_{j}=\left(\prod_{s=j}^{0} w_{s}\right)$ if $-q \leq j<1$. In particular, if $C:=\max \left\{C_{j}:|j| \leq q\right\}$ and $\delta<\frac{\epsilon}{C\left\|T^{2 q}\right\|}$, then we have $\prod_{s=0}^{n-1} w_{s+j}<\epsilon$ for all $j$ with $|j| \leq q$.

Also, with the same choice of $n$ (which guarantees that $n-j \geq n_{k}+1$ ) and $|j| \leq q$ we have

$$
\begin{aligned}
\prod_{s=1}^{n} \frac{1}{w_{j-s}} & =\prod_{s=1-j}^{n-j} \frac{1}{w_{-s}}=C_{j}^{\prime}\left(\prod_{s=1}^{n_{k}} \frac{1}{w_{-s}}\right) \prod_{s=n_{k}+1}^{n-j} \frac{1}{w_{-s}} \\
& \leq C_{j}^{\prime}\left(\frac{1}{m}\right)^{2 q}\left(\prod_{s=1}^{n_{k}} \frac{1}{w_{-s}}\right) \leq C_{j}^{\prime}\left(\frac{1}{m}\right)^{2 q} \delta
\end{aligned}
$$

where $m$ is a lower bound for the negative weights and $\left\{C_{j}^{\prime}\right\}$ is a finite collection of positive constants, depending only on $q$. Thus if we let $C^{\prime}=\max \left\{C_{j}^{\prime}\right\}$ and also require that $\delta<\frac{m^{2 q} \epsilon}{C^{\prime}}$, then we will have $\prod_{s=1}^{n} \frac{1}{w_{j-s}}<\epsilon$ as required.

Thus $T$ is hypercyclic by Theorem 2.3.

(2) Again we will verify Salas' condition in Theorem 2.4 So, let $\epsilon>0$ and $q \in \mathbb{N}$. Suppose $\delta>0$ (we will prescribe $\delta$ later); then there exists an (arbitrarily large) $n_{k}$ such that $\left(\prod_{j=1}^{n_{k}} w_{j}\right)\left(\prod_{j=1}^{n_{k}} \frac{1}{w_{-j}}\right)<\delta$. Now let $n=n_{k}+q+1$ and suppose that $j, h \in \mathbb{Z}$ and $|j| \leq q$ and $|h| \leq q$. Then using the estimates from part (1) above we 
have:

$$
\begin{gathered}
\left(\prod_{k=j}^{j+n-1} w_{k}\right)\left(\prod_{k=h-1}^{h-n} \frac{1}{w_{k}}\right)=\left(\prod_{k=j}^{j+n-1} w_{k}\right)\left(\prod_{k=1-h}^{n-h} \frac{1}{w_{-k}}\right) \\
\leq C_{j}\left\|T^{2 q}\right\| \delta\left(C_{h}^{\prime}\left(\frac{1}{m}\right)^{2 q} \delta\right) .
\end{gathered}
$$

Thus we have that $\left(\prod_{k=j}^{j+n-1} w_{k}\right)\left(\prod_{k=h-1}^{h-n} \frac{1}{w_{k}}\right) \leq K \delta^{2}$ where $K=C_{j} C_{h}^{\prime}\left\|T^{2 q}\right\| m^{-2 q}$ is a constant depending only on $q$. Thus, if $\delta$ is small enough, then we will have $K \delta^{2}<\epsilon$, hence Salas' condition is satisfied. Thus $T$ is supercyclic.

The proof is similar when the positive weights are bounded below and is left to the reader (in this case choose $n=n_{k}-q$ ).

We now apply our criteria to obtain a condition for the direct sum of two invertible bilateral weighted shifts to be hypercyclic or supercyclic. One should observe that the criterion given by Salas [8] for direct sums to be supercyclic is stated incorrectly.

Theorem 4.2. Suppose that $T_{1}$ and $T_{2}$ are invertible bilateral weighted shifts with weight sequences $\left(a_{n}\right)_{n=-\infty}^{\infty}$ and $\left(b_{n}\right)_{n=-\infty}^{\infty}$. Then:

(1) $T_{1} \oplus T_{2}$ is hypercyclic if and only if there exists a sequence of integers $n_{k} \rightarrow \infty$ such that

$$
\lim _{k \rightarrow \infty} \prod_{j=1}^{n_{k}} a_{j}=\lim _{k \rightarrow \infty} \prod_{j=1}^{n_{k}} \frac{1}{a_{-j}}=\lim _{k \rightarrow \infty} \prod_{j=1}^{n_{k}} b_{j}=\lim _{k \rightarrow \infty} \prod_{j=1}^{n_{k}} \frac{1}{b_{-j}}=0 .
$$

(2) $T$ is supercyclic if and only if there exists a sequence of integers $n_{k} \rightarrow \infty$ such that

$$
\lim _{k \rightarrow \infty}\left(\prod_{j=1}^{n_{k}} \frac{a_{j}}{a_{-j}}\right)=\lim _{k \rightarrow \infty}\left(\prod_{j=1}^{n_{k}} \frac{b_{j}}{b_{-j}}\right)=\lim _{k \rightarrow \infty}\left(\prod_{j=1}^{n_{k}} \frac{a_{j}}{b_{-j}}\right)=\lim _{k \rightarrow \infty}\left(\prod_{j=1}^{n_{k}} \frac{b_{j}}{a_{-j}}\right)=0 .
$$

The proof of the above result is similar to that of Theorems 3.2 and 3.4 and the details will be left to the reader.

\section{ACKNOWLEDGEMENTS}

The author would like to thank the referee for his/her comments and suggestions.

\section{REFERENCES}

[1] P. S. Bourdon and J. H. Shapiro, Cyclic Phenomena for Composition Operators, Memoirs of the AMS, 125, AMS, Providence, RI, 1997. MR 97h:47023

[2] N.S. Feldman, T.L. Miller and V.G. Miller Hypercyclic and Supercyclic Cohyponormal Operators, To appear in Acta. Sci. Math.

[3] R. M. Gethner and J. H. Shapiro, Universal vectors for operators on spaces of holomorphic functions, Proc. AMS, 100 (1987), 281-288. MR 88g:47060

[4] H. M. Hilden and L. J. Wallen, Some cyclic and non-cyclic vectors of certain operators, Ind. Univ. Math. J., 23 (1974), 557-565. MR 48:4796

[5] C. Kitai, Invariant closed sets for linear operators, Dissertation, Univ. of Toronto, 1982.

[6] S. Rolewicz, On orbits of elements, Studia Math., 32 (1969), 17-22. MR 39:3292 
[7] H. N. Salas, Hypercyclic weighted shifts, Trans Amer. Math. Soc., 347 (1995), 993-1004. MR 95e: 47042

[8] H. N. Salas, Supercyclicity and weighted shifts, Studia Math. 135 (1999), no. 1, 55-74. MR 2000b: 47020

Department of Mathematics, Washington and Lee University, Lexington, Virginia 24450

E-mail address: feldmann@wlu.edu 